\title{
Inclusion Phenomena between the $\beta$-Cyclodextrin Chiral Selector and Trp-D,L, and Its Use on the Assembly of Solid Membranes
}

\author{
Hong Meng, Sumin Li, Ling Xiao, and Chunxi Li \\ College of Chemical Engineering, Beijing University of Chemical Technology, Beijing 100029, China
}

Correspondence should be addressed to Hong Meng; menghong@mail.buct.edu.cn

Received 3 May 2013; Accepted 7 July 2013

Academic Editor: Hamed Bahmanpour

Copyright (C) 2013 Hong Meng et al. This is an open access article distributed under the Creative Commons Attribution License, which permits unrestricted use, distribution, and reproduction in any medium, provided the original work is properly cited.

\begin{abstract}
The application of $\beta$-cyclodextrin $(\beta$-CD) and the chiral ionic liquids formed from $\beta$-cyclodextrin mono-6-deoxy-6-(3methylimidazolium)- $\beta$-cyclodextrin tosylate $(\beta$-CD-IL) as chiral selectors is described. The inclusion phenomena between the $\beta$-cyclodextrin chiral selectors and D,L-tryptophan (D,L-Trp) was studied. The inclusion compounds were prepared by grinding, and their properties analyzed by X-ray diffraction (XRD), Fourier transform infrared spectroscopy (FT-IR), and nuclear magnetic resonance (NMR). The separation factor between $\beta$-CD and $\beta$-CD-IL with D,L-Trp was studied by the saturated solution method. This indicated a different binding capacity of $\beta$-CD and $\beta$-CD-IL to the two enantiomers. This result shows that the chiral ionic liquids have a higher separation factor because of their high solubility. The $\beta$-cyclodextrin chiral ionic liquids and CS were crosslinked and immobilized on an N6 membrane to form composite membranes. Adsorption experiments and permeation experiments were carried out. $105.43 \mathrm{mg}$ D,L-Trp/g membrane was obtained.
\end{abstract}

\section{Introduction}

Chiral separation is an important process used in both the pharmaceutical and chemical industries [1]. There are many traditional methods to separate enantiomers from the racemic mixture [2] but the membrane separation process is a newly emerging technology that shows good prospects. Liquid membrane and solid membrane enantiomer separation methods have been extensively studied $[3,4]$. Solid membrane separation is considered to have many advantages, such as long term stability and low energy consumption, and it is easy to scale up [5]. For these reasons it has developed rapidly.

A chiral solid membrane is a type of chiral recognition agent prepared using blending and coating methods. The result is fixed in the polymer membrane. The chiral solid membrane is then used in a separation process to achieve the enantiomeric separation. Clearly, the chiral selector is one of the core technologies. Many substances can be used as chiral selectors, including proteins, antibiotics, polysaccharides, amino acids, apo-enzymes, DNA, and surfactant supramolecular compounds (such as cyclodextrins and crown ethers) [6]. For example, Singh et al. prepared the composite membranes by the interfacial polymerization of 1 -arginine and piperazine with trimesoyl chloride in situ on microporous polysulfone membrane. They pointed out that the enantioselectivity of membrane has occurred due to the interaction of permeating isomers to chiral centers present on the top layer of the membrane [7]. More recently, Singh et al. also fabricated $\beta$ cyclodextrin glutaraldehyde cross-linked polysulfone membrane by phase inversion technique. The experimental results indicated that incorporation of $\beta$-cyclodextrin in polysulfone polymer had provided enantiomer discriminating capability to the membrane; therefore, $\beta$-CDXM had exhibited enantioselectivity for D-Phe and D-Trp. The observation confirmed that the chiral environment is essential for enantiomer separation [8]. Iritani et al. accomplished enantiomeric separation of tryptophan with BSA as the stereospecific macroligand by means of affinity ultrafiltration of single-pass mode using hollow fiber membrane module. It was found that the 
separation factor of d-tryptophan in affinity ultrafiltration was increased with the increase in the BSA concentration and the decrease of the racemic tryptophan concentration due to preferentially permeation of unbound d-tryptophan through the membrane at $\mathrm{pH} 7$ [9].

Various chiral selectors, such as cyclodextrins, molecular micelles, antibodies, and crown ethers, have been widely used because of their chiral recognition abilities [10]. These are capable of selectively complexing with guest molecules by virtue of a series of weak intermolecular forces. This technique has been used to produce chiral selectors for many years [11]. This type of selector always forms nonconducting compounds with the guest molecule. They can also be used as chiral agents during membrane separation processes. However, there are some difficulties to be solved. For instance, $\beta$-CD has poor solubility, which limits its use, although improvements in solubility are being developed. Derivatives of $\beta$-CD can be prepared that would enhance its inclusion ability $[12,13]$. When these have been used on membranes, the separation factor has been improved.

A chiral ionic liquid is a kind of chiral selector with the advantages of an ionic liquid such as low vapor pressure, high solubility, and high stability at high temperature. It can be used simultaneously as the solvent and chiral selector. Thus, the use of chiral ionic liquids has gained popularity, since they can be used as chiral solvents for asymmetric induction in synthesis [14]. They can also be immobilized on the solid membrane where they show chiral separation ability at the same time as high inclusion ability [15]. This kind of chiral recognition has received increasing attention, recently. For example, Tang et al. reported that functional amino acid ionic liquids (AAILs) could be used as solvent and selector in chiral liquid-liquid extraction. In their work, using these functional AAILs as acceptor phase and ethyl acetate as donor phase, more l-enantiomer of amino acid was extracted into the ionic-liquid phase than that of d-enantiomer [16]. Bi et al. developed a simple and accurate method for the separation and determination of ofloxacin enantiomers by ionic liquid-assisted ligandexchange high performance liquid chromatography. Comparing achiral ILs with chiral ILs, the latter offered superior enantioseparation efficiency, with [BMIM]-[Leu] the leader among them [17]. Absalan et al. found that the chiral ionic liquid 1-butyl-3-methylimidazolium (T-4)-bis[(aS)-a(hydroxy-O)benzeneacetate-kO] borate was an appropriate chiral recognizing agent for propranolol hydrochloride enantiomers by using the UV-vis spectrophotometric technique. In comparison to other chiral selectors that have been used for determination of propranolol, chiral ionic liquids are more favorable as their synthesis is simple, inexpensive, and they are friendly for environmental applications [18]. More recently, Yu et al. synthesized a novel chiral ionic liquid functionalized b-cyclodextrin,6-O-2hydroxypropyltrimethylammonium-b-cyclodextrin tetrafluoroborate ([HPTMA-b-CD] $\left.\left[\mathrm{BF}_{4}\right]\right)$, which was used as a chiral selector in capillary electrophoresis. It was found that [HPTMA-b-CD] $\left[\mathrm{BF}_{4}\right]$ not only increased the solubility in aqueous buffer in comparison with the parent compound but also provided a stable reversal electroosmotic flow. Moreover, the chiral ILs were applied for the enantiomer separation of eight racemic drugs (chlorpheniramine, brompheniramine, pheniramine, Tropicamide, Bifonazole, promethazine, warfarin, and liarozole) by capillary electrophoresis [19].

Chitosan (CS) has desirable membrane-forming ability together with excellent biocompatibility and good hydrophilicity, which makes it popular for applications in membrane-mediated separation processes $[20,21]$. Due to the presence of a large number of chiral sites, CS exhibits excellent chiral selectivity [22].

In this paper, we describe the synthesis of the chiral ionic liquid mono-6-deoxy-6-(3-methylimidazolium)- $\beta$ cyclodextrin tosylate. Its solubility properties were studied. The inclusion phenomena was observed between the $\beta$ cyclodextrin chiral selector and D,L-Trp using a grinding method. Other properties of the compounds were analyzed by XRD, FT-IR, and NMR. The separation factor between $\beta$-CD and $\beta$-CD-IL with Trp-D,L was measured using the saturated solution method and indicated different binding capacities for $\beta$-CD and $\beta$-CD-IL to the enantiomers. The experimental results show that the chiral ionic liquid had a higher separation factor because of its high solubility. The $\beta$-cyclodextrin chiral ionic liquids and CS were cross-linked and immobilized on the N6 membrane to form composite membranes. Adsorption experiments and permeation experiments were carried out [23].

\section{Materials and Methods}

2.1. Materials. D,L-tryptophan (D,L-Trp), D-tryptophan (DTrp), and L-tryptophan (L-Trp) were obtained from Shanghai Crystal Pure Reagent Co., Ltd. Beta-cyclodextrin ( $\beta$-CD), methyl-benzenesulfonyl chloride, formic acid, sulfonylurea chloride, sodium hydroxide acetonitrile, hydrochloric acid, potassium chloride, N, N-DMF (DMF), acetone, glutaraldehyde (GA, 25 wt.\% aqueous solution), and acetic acid were provided by the Beijing Chemical Factory. Nylon-6 (N6) substrate membrane ( $95 \mu \mathrm{m}$ thick), with an average pore size of $0.22 \mu \mathrm{m}$, was purchased from Zhejiang Xidoumen Membrane Co.

\subsection{Preparation of Inclusion Compounds and Mechanical} Mixing. The inclusion compounds formed between $\beta-\mathrm{CD}$ and $\beta$-CD-IL with D,L-Trp were prepared using the grinding method [24]. A small amount of deionized water was added to a mortar with $2.27 \mathrm{~g} \beta$-CD (or $3.07 \mathrm{~g} \beta$-CD-IL). The $\beta$ $\mathrm{CD}$ (or $\beta$-CD-IL) was ground, D,L-Trp was added and mixed to form a paste (molar ratio chiral selector: D,L-Trp $=1: 1$ ). The paste was dried at $40^{\circ} \mathrm{C}$ for $6 \mathrm{~h}$. It was then washed with deionized water and dried to give the final product. The mixtures of $\beta$-CD or $\beta$-CD-IL with D,L-Trp were prepared at the same time. The raw materials were weighed to give a molar ratio of $1: 1$ and mixed using a spatula until a homogeneous mixture was obtained.

2.3. X-Ray, FT-IR, and NMR Analyses. X-ray diffraction data for the inclusion compounds and mixtures were obtained with a multifunctional 2500VB2 + PC X-ray diffractometer 
(Kabushiki Kaisha, Japan) using $\mathrm{Ni}$-filtered, $\mathrm{Cu} \mathrm{K} \alpha$ radiation, a voltage of $40 \mathrm{kV}$, and a $30 \mathrm{~mA}$ current. Analyses were performed on the same samples prepared for DSC studies. All samples were measured in the 2 theta angle range between $10^{\circ}$ and $80^{\circ}$ with a scan rate of $8^{\circ} / \mathrm{min}$ and a step size of $0.02^{\circ}$. All samples were analysed in triplicate. The infrared spectra (FTIR) of the catalysts were recorded on a Nicolet Nexus 8700 FT-IR spectrometer as $\mathrm{KBr}$ disks over the range $400-4000 \mathrm{~cm}^{-1}$. NMR analysis was carried out on an NMR spectrometer (AV600) from Bruker [25].

2.4. The Saturated Solution Method. $\beta$-CD (or $\beta$-CD-IL) was dissolved in $100 \mathrm{~mL}$ deionized water until a saturated solution was obtained. D,L-Trp was completely dissolved in a small quantity of formic acid. This solution was then added to the saturated solutions of $\beta$-CD (or $\beta$-CD-IL) to give molar ratios of $\beta$-CD (or $\beta$-CD-IL): D,L-Trp ranging from 1 to 5 (or 35). The mixtures were stirred for $48 \mathrm{~h}$ in the dark at a defined temperature, left in the refrigerator at $4^{\circ} \mathrm{C}$ for $24 \mathrm{~h}$, then filtered to remove the precipitate. The resulting solution was diluted with deionized water to give $1 \mathrm{~L}$. The concentration of D,L-Trp in the solution was analysed by HPLC, and the separation factor was obtained, as described by Song et al. [26].

\subsection{Preparation of Chitosan/ $\beta$-Cyclodextrin Composite Mem-} branes. The chiral ionic liquid $\beta$-CD-IL was synthesized according to previously published papers [27]. The process for the preparation of the CS/CD composite membranes was as follows. $1.0 \mathrm{~g}$ of CS was dissolved in $50 \mathrm{~mL}$ of $2 \mathrm{wt} . \%$ aqueous acetic acid solution. After the removal of impurities, a defined amount of $\beta$-CD-IL was added, with stirring. The suspension was then agitated for $12 \mathrm{~h}$ at room temperature to guarantee an even suspension mixture. Afterwards, $0.2 \mathrm{~mL}$ of GA was added in order to cross-link the CS and then the suspension was further agitated for another $20 \mathrm{~min}$. The N6 membrane substrate, already saturated with deionized water, was immersed in the suspension, and the subsequent mixture was left to stand for $1 \mathrm{~h}$. At the end of the immersion process, the membrane was removed and spread on a flat and clean glass plate after carefully removing the redundant casting solution on the membrane surface in contact with the glass plate. The membrane was dried at $50^{\circ} \mathrm{C}$ for $8 \mathrm{~h}$ to form the CS/CD semi-IPN (interpenetrating network) layer on the N6 substrate. This was then washed alternately with 1.0 M aqueous $\mathrm{NaOH}$ solution and deionized water. Finally, the prepared CS/CD composite membrane was again dried at $50^{\circ} \mathrm{C}$ overnight.

2.6. Sorption Experiments. The dry membrane specimens were immersed in aqueous $\operatorname{Trp}$ racemate solutions with a concentration of $1 \mathrm{mg} / \mathrm{mL}$ at $25^{\circ} \mathrm{C}$ for $48 \mathrm{~h}$ to reach sorption equilibrium. Afterwards, the D- and L-Trp concentrations in the solutions were analysed by HPLC to determine the

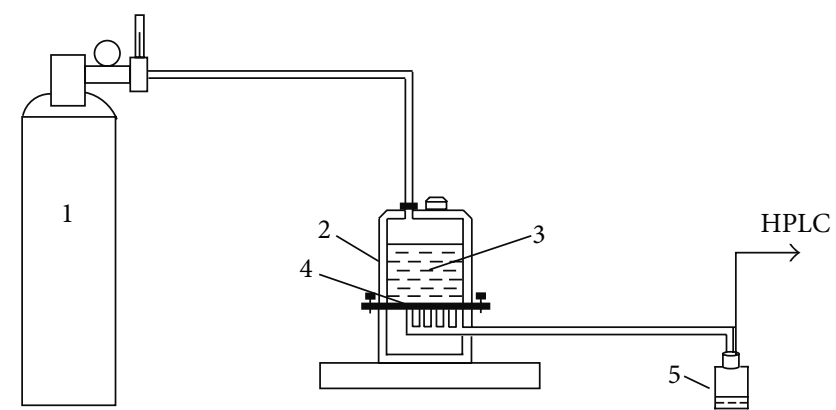

FIgURE 1: The experimental apparatus. (1) Nitrogen cylinder, (2) ultrafiltration cup, (3) tryptophan (D,L-Trp) racemate solution, (4) chiral solid membrane, (5) meet liquid bottle.

sorption selectivity and adsorbed Trp content. These are expressed by (1)

$$
\begin{gathered}
\alpha S=\frac{\left(C_{d_{i}}-C_{d_{f}}\right) / C_{d_{f}}}{\left(C_{l_{i}}-C_{l_{f}}\right) / C_{l_{f}}}=\frac{\left(C_{d_{i}}-C_{d_{f}}\right) /\left(C_{l_{i}}-C_{l_{f}}\right)}{C_{d_{f}} / C_{l_{f}}}, \\
Q=\frac{\left(C_{i}-C_{f}\right) V}{m},
\end{gathered}
$$

where $C_{d_{i}}$ and $C_{d_{f}}$ denote the initial and final concentrations of D-Trp and $C_{l_{i}}$ and $C_{l_{f}}$ denote the initial and final concentrations of L-Trp, respectively. $C_{i}$ and $C_{f}$ represent the initial and final Trp concentrations in the solution, $V$ the solution volume, and $m$ the weight of dry membrane. A sorption experiment was carried out to measure the sorption selectivity and sorption ability of CS/CD composite membranes, -CDP, and chitosan. Due to the fact that the amount of adsorbed 1-Trp was always relatively large compared with that of $\mathrm{d}$-Trp, the value $\alpha S$ was always lower than unity so a relatively low $\alpha S$ indicated a relatively high sorption selectivity. Identical amounts of -CDP and chitosan flakes (10 mg of each) were suspended in aqueous Trp racemate solution with a concentration of $1.0 \mathrm{mg} / \mathrm{mL}$ at $25^{\circ} \mathrm{C}$ for $48 \mathrm{~h}$ to reach sorption equilibrium. The suspensions were centrifuged and then the supernatant was subjected to HPLC analysis. The determination of sorption selectivity and adsorbed Trp content was the same as that for the membranes.

2.7. Ultrafiltration Separation Experiment. Chiral separation experiments were carried out at room temperature in a laboratory scale filtration unit, as shown in Figure 1.

This contains a cross-flow permeation cell with an effective filtration area of $38.47 \mathrm{~cm}^{2}$ supported by a porous stainless steel disc. The transmembrane pressure can be controlled over the range $0-0.1 \mathrm{MPa}$.

The L-tryptophan and D-tryptophan feed solutions were prepared by dissolving them in pure water at a concentration of about $0.1 \mathrm{~g} / \mathrm{L}$. Before the penetrant was collected, the accurate concentrations of tryptophan and IL-CD were analysed by HPLC. 
The apparent separation factor $\alpha$ was calculated using the following equation:

$$
\alpha=\frac{\left(C_{p_{(D)}}\right) /\left(C_{p_{(L)}}\right)}{\left(C_{f_{(D)}}\right) /\left(C_{f_{(L)}}\right)},
$$

where $C_{p_{(D)}}$ and $C_{p_{(L)}}$ are the D-Trp and L-Trp $(\mathrm{g} / \mathrm{L})$ solute concentrations in the penetrant, respectively. $C_{f_{(D)}}$ and $C_{f_{(L)}}$ are the D-Trp and L-Trp $(\mathrm{g} / \mathrm{L})$ solute concentrations in the feed solution.

The penetration flux $Q$ was calculated using the following equation:

$$
Q=\frac{q}{A \times t} .
$$

The concentration polarization can be ignored since a high feed velocity across the membrane surface was applied. Each separation experiment was repeated three times and an average was calculated for further analysis.

2.8. High-Performance Liquid Chromatography (HPLC) Analyses. High-performance liquid chromatographic analysis was carried out using an external standard method on a HPLC apparatus equipped with a Daicel CROWNPAK CR(+) column $(150 \mathrm{~mm} \times 4 \mathrm{~mm}$ I.D. $)$ and a UV detector $(280 \mathrm{~nm})$. Chiral analysis was performed using a mobile phase containing perchloric acid $(\mathrm{pH}=2.0) /$ methanol $(86: 14, \mathrm{v} / \mathrm{v})$. The flow rate was $1.5 \mathrm{~mL} / \mathrm{min}$. All the chiral analyses were performed in triplicate.

\section{Results and Discussion}

3.1. XRD Diffraction Analysis. Powder X-ray diffraction is a useful method for the detection of CD complexation in the powder or microcrystalline states. The diffraction pattern of the complex is often clearly distinct from that of the superposition of each of the components, if a true inclusion complex is formed [28]. The XRD diffraction patterns of $\mathrm{D}, \mathrm{L}-\mathrm{Trp}, \beta-\mathrm{CD}$, the mechanical mixture, $\mathrm{D}, \mathrm{L}-\operatorname{Trp}$, and the $\beta$ CD inclusion complex are presented in Figure 2. The XRD pattern of D,L-Trp showed intense, sharp peaks that prove the crystalline nature of the compound. D,L-Trp had strong peaks at 2 theta values of $9^{\circ}, 15^{\circ}, 19^{\circ}$, and several minor peaks around $29^{\circ}$ and $35^{\circ}$. On the other hand, the XRD pattern of $\beta$-CD revealed several peaks in the range $8^{\circ}-34^{\circ}$, confirming its amorphous character. In the case of D,L-Trp and the $\beta$-CD mechanical mixture with a molar ratio of $1: 1$, the diffraction pattern was simply the superposition of the two patterns of the crystalline D,L-Trp and the amorphous HP-b-CD. However, the inclusion complex of D,L-Trp with $\beta$-CD gave a different pattern from the mechanical mixture, particularly at peaks (a), (b), (c), and (d), (Figure 3) which indicates the formation of the inclusion of a D,L-Trp with $\beta$ CD.

As shown in Figure 3, the XRD pattern of -IL showed broad peaks in the range $10^{\circ}-25^{\circ}$, confirming its amorphous character, which is different from the pattern given by $\beta$ CD. The pattern of the mechanical mixture is clearly the

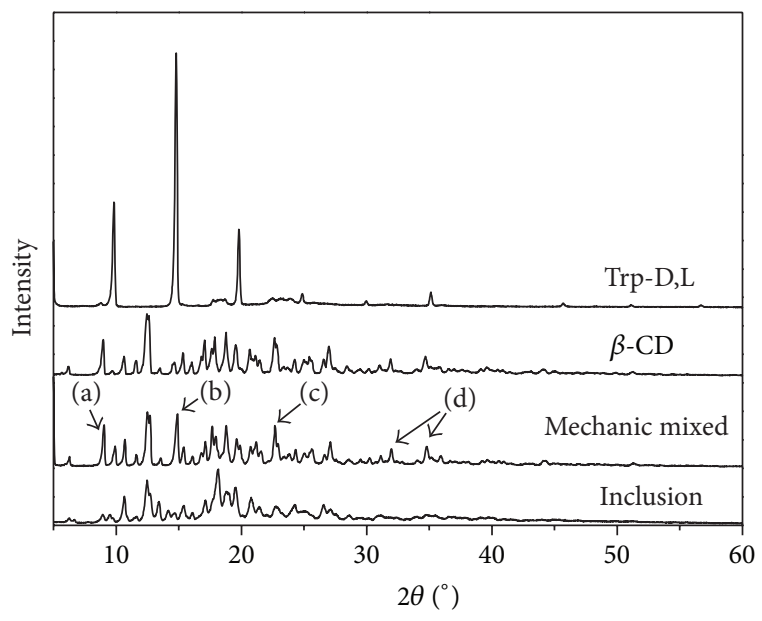

Figure 2: Power X-ray diffraction patterns of D,L-Trp, $\beta$-CD, mechanic mixture, $\mathrm{D}, \mathrm{L}-\mathrm{Tr}$, and $\beta$-CD inclusion.

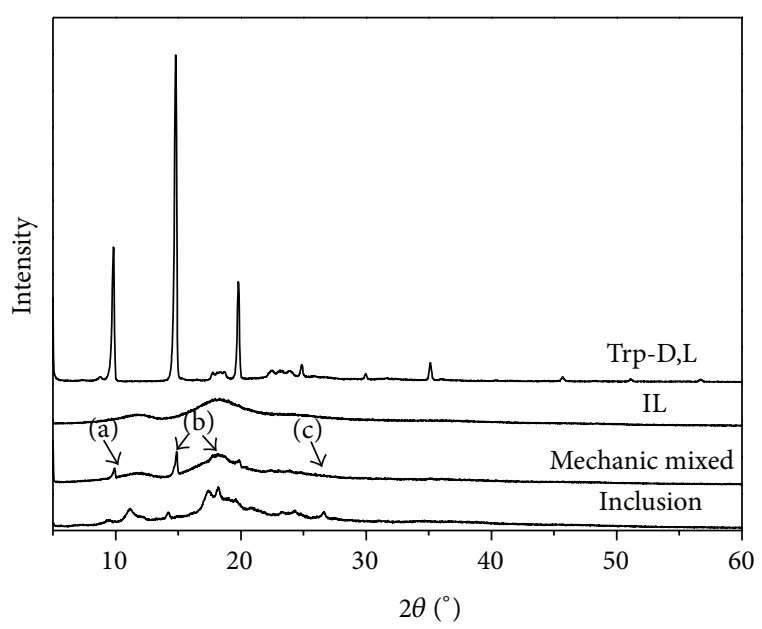

Figure 3: Power X-ray diffraction patterns of D,L-Trp, IL, mechanic mixed, and D,L-Trp-IL inclusion.

superposition of the patterns of the two components. In this case the molar ratio of D,L-Trp and $\beta$-CD is $1: 1$. At peaks (a), (b), and (c) of Figure 3, the inclusion compounds produced a different pattern, which indicates the formation of the inclusion complex of D,L-Trp with $\beta$-CD.

3.2. FT-IR Diffraction Analysis. FT-IR is a technique frequently used to indicate the formation of an inclusion complex. The FT-IR spectra of D and L-Trp, $\beta$-CD, the mechanical mixture, and $\mathrm{D}$ and L-Trp with the $\beta$-CD inclusion complex are presented in Figure 4. The molar ratio of D,L-Trp and $\beta$-CD is $1: 1$. The pattern of the mechanical mixture is obviously the superposition of the patterns of the two components. This shows absorption peaks at (a) $\left(3404.3 \mathrm{~cm}^{-1}\right)$, (b) $\left(3079.8 \mathrm{~cm}^{-1}\right)$, and (c) $\left(1593.1 \mathrm{~cm}^{-1}\right)$ which belong to the characteristic peaks of D and L-Trp. The absorption peaks of the inclusion complex do not show any obvious sharp peaks, which indicates that the inclusion complex is not the same 


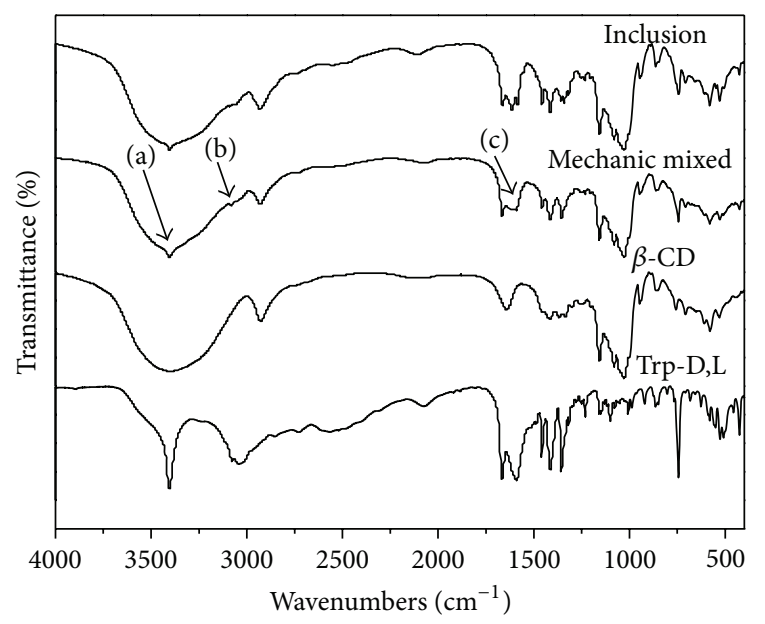

FIgURE 4: FT-IR spectra of Trp-D,L, $\beta$-CD, mechanic mixed, and Trp-D,L- $\beta$-CD inclusion.

substance as the mechanical mixture. The absorption peaks of the inclusion complex turn out to be narrow, indicating that the inclusion compound is not a mechanical mixture of the $\mathrm{D}, \mathrm{L}-\mathrm{Trp}$ and $\beta$-CD but a new material, demonstrating that the inclusion reaction has occurred.

3.3. NMR Analysis. The NMR spectra shows the chemical shifts of hydrogen atoms, carbon atoms, and size, which inferred the formation of inclusion complexes. ${ }^{1} \mathrm{H}$ NMR techniques can generally be used to analyze the inclusion phenomenon when the substances contain aromatic moieties. The FT-IR spectra of D and L-Trp, $\beta$-CD-IL, the mechanical mixture, and $\mathrm{D}$ and L-Trp with the $\beta$-CD-IL inclusion complex are presented in Figures 5, 6, 7, and 8. Figures 5, 7, and 9 contrast the inclusion product generated from the two original substances $\mathrm{D}$ and L-Trp with $\beta$-CD-IL. The spectra of the ionic liquids show significant differences, which indicate that after the inclusion reaction new material is generated. The difference between Figures 7 and 8 is clear. The inclusion complex is a different material.

3.4. The Results from the Saturated Solution Method. The reaction between D,L-Trp and $\beta$-CD was carried out, with the result shown in Tables 1 and 2. According to the HPLC analysis, D-tryptophan and L-tryptophan are both reduced after the reaction, which demonstrates the formation of the inclusion complex between $\beta$-CD (or $\beta$-CD-IL) and D,L-Trp. From the experimental observation, we know that the more $\beta$-CD (or $\beta$-CD-IL) is added the more inclusion compound is generated. Under the same conditions, $\beta$-CD-IL with higher solubility could bond to more guest molecules and show better inclusion ability.

Because of the low solubility of $\beta-\mathrm{CD}$, the reaction with $\beta$-CD-IL was possible even with the large molar ratio of $35: 1$, reflecting the advantages of $\beta$-CD-IL, which is more energy efficient than $\beta$-CD.

3.5. Sorption Experiments and Ultrafiltration Separation of $D$ and L-Trp. It can be seen from Tables 3 and 4 that the
${ }^{1} \mathrm{H}$ NMR of Trp in DMSO-d6, $400 \mathrm{MHz}$, at $300 \mathrm{~K}$

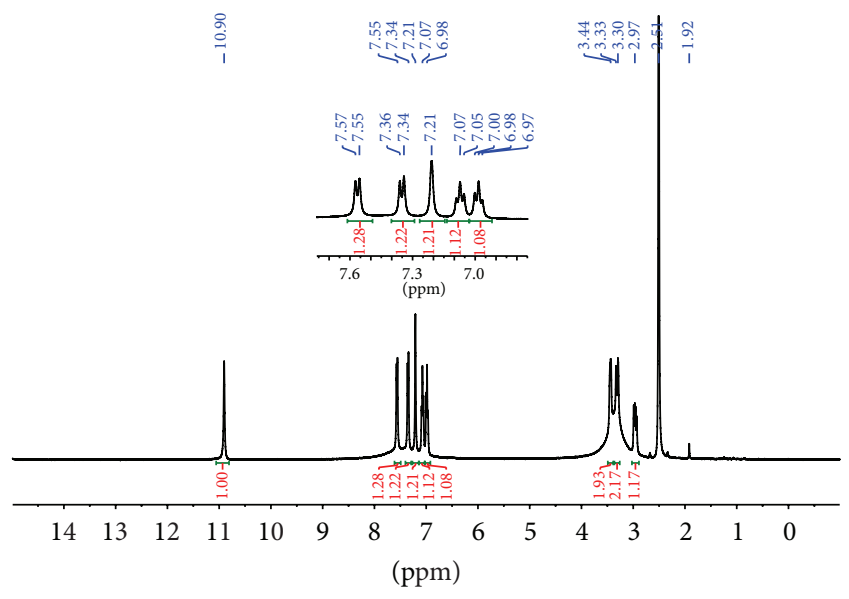

Figure 5: ${ }^{1} \mathrm{H}$ NMR of D,L-Trp.

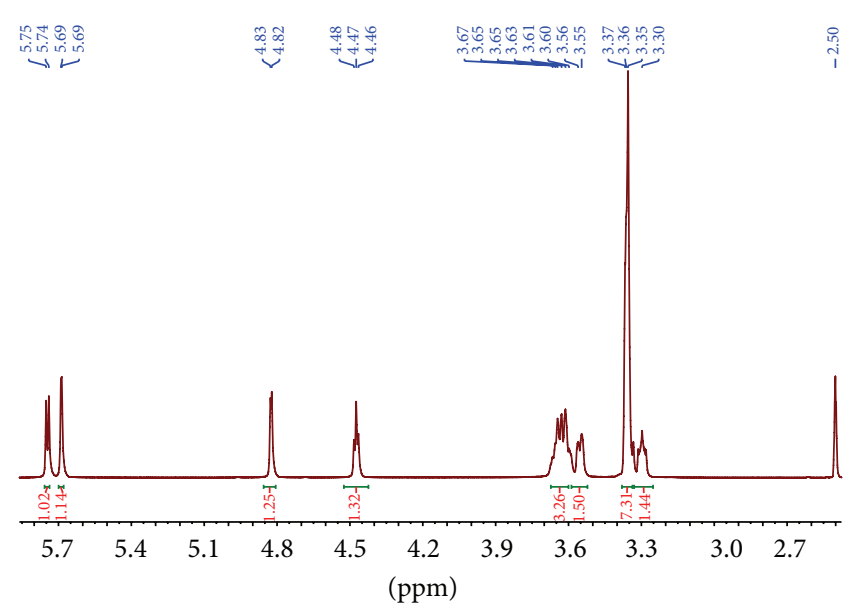

Figure $6:{ }^{1} \mathrm{H}$ NMR of $\beta$-CD.

TABLE 1: Inclusion reaction result of D,L-Trp and $\beta$-CD.

\begin{tabular}{|c|c|}
\hline$\beta$-CD : D,L-Trp (molar ratio) & Split factor \\
\hline $1: 1$ & 1.1 \\
\hline
\end{tabular}

TABLE 2: Inclusion reaction result of D,L-Trp and $\beta$-CD-IL.

\begin{tabular}{lc}
\hline$\beta$-CD-IL:D,L-Trp (molar ratio) & Split factor \\
\hline $1: 1$ & 1.1 \\
$5: 1$ & 1.1 \\
$20: 1$ & 1.2 \\
$25: 1$ & 1.3 \\
$30: 1$ & 1.3 \\
$35: 1$ & 1.4 \\
\hline
\end{tabular}

chiral solid membrane shows chiral recognition features. The CS membrane gave higher chiral selectivity than the membranes after cross-linking with $\beta$-CD-IL. The selectivity was reduced as the proportion of $\beta$-CD-IL increased. This 
TABLE 3: Sorption experiment results.

\begin{tabular}{lc}
\hline Membrane & Sorption ability (mg/g membrane) \\
\hline CS (1 g) & 3.4 \\
CS/IL (1 g:0.3 g) & 14.8 \\
CS/IL (2 g:0.6 g) & 47.3 \\
CS/IL (2 g:1.2 g) & 105.5 \\
\hline
\end{tabular}

TABLE 4: Ultrafiltration separation results.

\begin{tabular}{lc}
\hline Membrane & Spit factor \\
\hline CS $(1 \mathrm{~g})$ & 1.2 \\
CS/IL (1 g:0.3 g) & 1.2 \\
CS/IL (2 g:0.6 g) & 1.5 \\
CS/IL (2 g:1.2 g) & 1.7 \\
\hline
\end{tabular}

may be due to the larger cavity of $\beta$-CD compared to the Trp molecule. However, the membrane without $\beta$-CD-IL showed lower selectivity, which indicates that the $\beta$-CD-IL improves chiral separation. In addition, the sorption ability became higher when $\beta$-CD-IL was added, which shows that the $\beta$-CD-IL aids the adsorption of tryptophan. The experiments were repeated with double the amount of agent in the casting solution. The results showed that the adsorption capacity was higher with the increase of the chiral recognition content within the membranes. In other words, increasing the content of $\beta$-CD-IL increases the adsorption capacity of the membrane.

In summary, XRD, FT-IR, and NMR analyses confirmed that an inclusion complex formed between $\beta$-CD-IL and tryptophan. Compared with the $\beta$-CD, $\beta$-CD-IL showed higher inclusion ability due to its higher solubility. Moreover, it was found that the $\beta$-CD-IL improves chiral separation when it was assembled onto solid membrane.

\section{Conclusions}

Chiral selectors are important for enantiomer separation. In this paper, mono-6-deoxy-6-(3-methylimidazolium)- $\beta$ cyclodextrin tosylate was prepared to improve the application of $\beta$-cyclodextrin for chiral separation. $\beta$-cyclodextrins usually generate inclusion compounds with guest molecules. The inclusion phenomena between $\beta$-cyclodextrin chiral selectors and D,L-Trp was studied. The inclusion compounds were prepared using a grinding method and their properties studied by XRD, FT-IR and NMR. The results indicate that the inclusion reaction had occurred. The separation factor between $\beta$-CD and $\beta$-CD-IL with D,L-Trp was studied by the saturated solution method and showed different binding capacities for $\beta$-CD and $\beta$-CD-IL to the enantiomers. The experiment revealed that the chiral ionic liquid had a higher separation factor because of its high solubility. The $\beta$-cyclodextrin/chitosan and $\beta$-cyclodextrin ionic liquids/chitosan composite membranes were prepared by casting methods. The influence of the content of $\beta$-cyclodextrin ionic liquid was investigated. A sorption experiment and

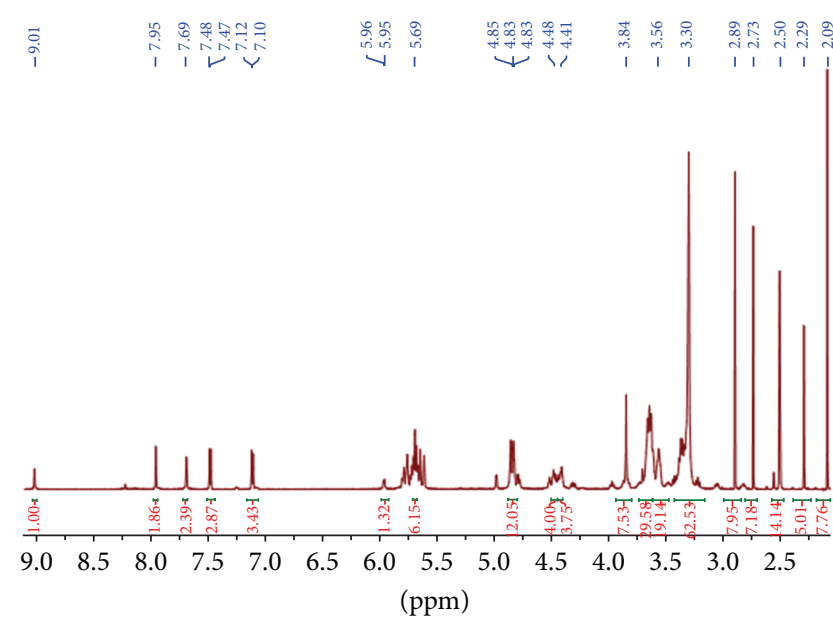

Figure 7: ${ }^{1} \mathrm{H}$ NMR of mono-6-deoxy-6-(3-methylimidazolium)- $\beta$ cyclodextrin tosylate.

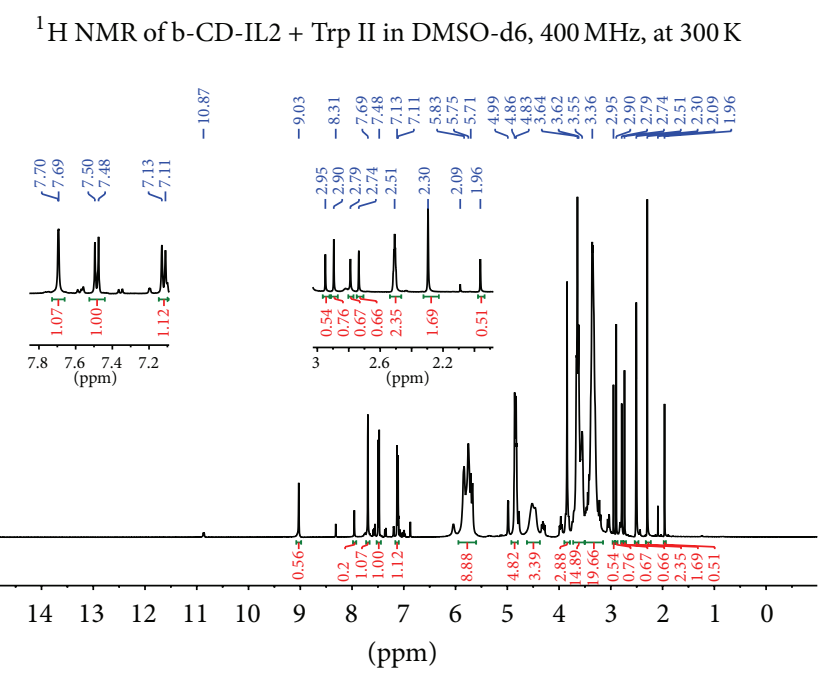

FIGURE 8: ${ }^{1} \mathrm{H}$ NMR of the physical mixture of mono-6-deoxy-6-(3methylimidazolium)- $\beta$-cyclodextrin tosylate and Trp.

an ultrafiltration separation experiment were carried out. The selectivity was reduced as the $\beta$-CD-IL content within the membranes increased, while the sorption selectivity increased. Chiral ionic liquids and CS were cross-linked and immobilized on the N6 membrane to form composite membranes. When the amount of agent in the casting solution doubled, the adsorption ability became higher. The results of this study provide information about the process of chiral separation and the potential for further improvements.

\section{Conflict of Interests}

The authors declared that the paper does not have any financial relation with the commercial identities mentioned in this paper. 
${ }^{1} \mathrm{H}$ NMR of b-CD-IL2-Trp I in DMSO-d6, $400 \mathrm{MHz}$, at $300 \mathrm{~K}$

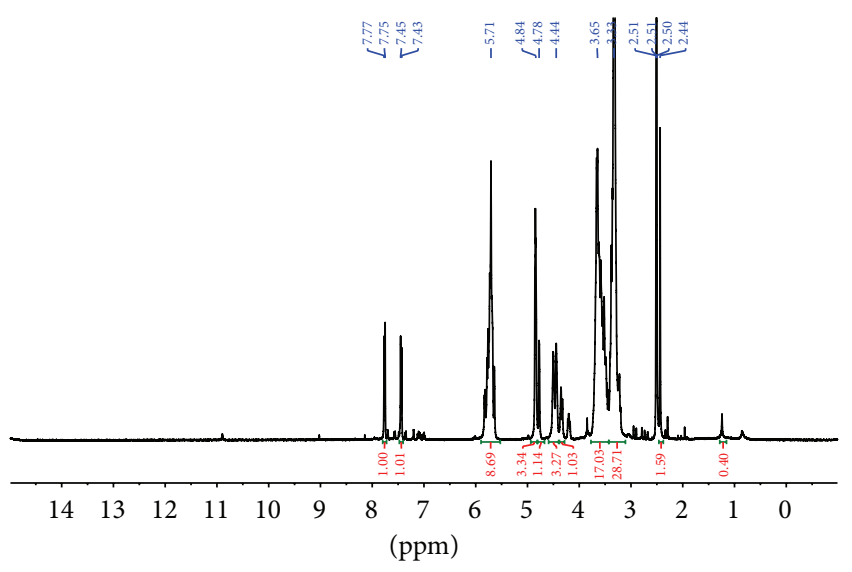

FIGURE 9: ${ }^{1} \mathrm{H}$ NMR of inclusion complex of mono-6-deoxy-6-(3methylimidazolium)- $\beta$-cyclodextrin tosylate and Trp.

\section{Acknowledgments}

The project was supported by the National Natural Science Foundation of China (program no. 21076010) and the Fundamental Research Funds for the Central Universities (program no. 221105).

\section{References}

[1] N. M. Maier, P. Franco, and W. Lindner, "Separation of enantiomers: needs, challenges, perspectives," Journal of Chromatography $A$, vol. 906, no. 1-2, pp. 3-33, 2001.

[2] Z. J. Li and D. J. W. Grant, "Relationship between physical properties and crystal structures of chiral drugs," Journal of Pharmaceutical Sciences, vol. 86, no. 10, pp. 1073-1078, 1997.

[3] M. Pietraszkiewicz, M. Koźbiał, and O. Pietraszkiewicz, "Chiral discrimination of amino acids and their potassium or sodium salts by optically active crown ether derived from D-mannose," Journal of Membrane Science, vol. 138, no. 1, pp. 109-113, 1998.

[4] A. Maximini, H. Chmiel, H. Holdik, and N. W. Maier, "Development of a supported liquid membrane process for separating enantiomers of N-protected amino acid derivatives," Journal of Membrane Science, vol. 276, no. 1-2, pp. 221-231, 2006.

[5] E. M. van der Ent, K. Van't Riet, J. T. F. Keurentjes, and A. van der Padt, "Design criteria for dense permeation-selective membranes for enantiomer separations," Journal of Membrane Science, vol. 185, no. 2, pp. 207-221, 2001.

[6] J. Romero and A. L. Zydney, "Chiral separations using ultrafiltration with a stereoselective binding agent," Separation Science and Technology, vol. 36, no. 7, pp. 1575-1594, 2001.

[7] K. Singh, P. G. Ingole, H. Bhrambhatt, A. Bhattachayra, and H. C. Bajaj, "Preparation, characterization and performance evaluation of chiral selective composite membranes," Separation and Purification Technology, vol. 78, no. 2, pp. 138-146, 2011.

[8] K. Singh, P. G. Ingole, H. C. Bajaj, and H. Gupta, "Preparation, characterization and application of $\beta$-cyclodextringlutaraldehyde crosslinked membrane for the enantiomeric separation of amino acids," Desalination, vol. 298, pp. 13-21, 2012.
[9] E. Iritani, N. Katagiri, T. Kawabata, and Y. Takaishi, "Chiral separation of tryptophan by single-pass affinity inclined ultrafiltration using hollow fiber membrane module," Separation and Purification Technology, vol. 64, no. 3, pp. 337-344, 2009.

[10] S. Fanali, "Enantioselective determination by capillary electrophoresis with cyclodextrins as chiral selectors," Journal of Chromatography A, vol. 875, no. 1-2, pp. 89-122, 2000.

[11] E. Schneiderman and A. M. Stalcup, "Cyclodextrins: a versatile tool in separation science," Journal of Chromatography B, vol. 745, no. 1, pp. 83-102, 2000.

[12] M. D. C. K. V. Ramos, L. H. P. Teixeira, F. R. de Aquino Neto, E. J. Barreiro, C. R. Rodrigues, and C. A. M. Fraga, "Chiral separation of $\gamma$-butyrolactone derivatives by gas chromatography on 2,3-di-O-methyl-6-O-tert-butyldimethylsilyl$\beta$-cyclodextrin," Journal of Chromatography A, vol. 985, no. 1-2, pp. 321-331, 2003.

[13] A. Shitangkoon, J. Yanchinda, and J. Shiowatana, "Thermodynamic study on the gas chromatographic separation of the enantiomers of aromatic alcohols using heptakis(2,3-diO-methyl-6-O-tert-butyldimethylsilyl)- $\beta$-cyclodextrin as a stationary phase," Journal of Chromatography A, vol. 1049, no. 1-2, pp. 223-226, 2004.

[14] C. Baudequin, J. Baudoux, J. Levillain, D. Cahard, A. Gaumont, and J. Plaquevent, "Ionic liquids and chirality: opportunities and challenges," Tetrahedron Asymmetry, vol. 14, no. 20, pp. 3081-3093, 2003.

[15] D. K. Bwambok, H. M. Marwani, V. E. Fernand et al., "Synthesis and characterization of novel chiral ionic liquids and investigation of their enantiomeric recognition properties," Chirality, vol. 20, no. 2, pp. 151-158, 2008.

[16] F. Tang, Q. L. Zhang, D. D. Ren, Z. Nie, Q. Liu, and S. Z. Yao, "Functional amino acid ionic liquids as solvent and selector in chiral extraction," Journal of Chromatography A, vol. 1217, no. 28, pp. 4669-4674, 2010.

[17] W. T. Bi, M. L. Tian, and K. H. Row, "Chiral separation and determination of ofloxacin enantiomers by ionic liquid-assisted ligand-exchange chromatography," Analyst, vol. 136, no. 2, pp. 379-387, 2011.

[18] G. Absalan, Y. Alipour, Z. Rezaei, and M. Akhond, "Determination of enantiomer compositions of propranolol enantiomers by chiral ionic liquid as a chiral selector and the UV-assisted spectrophotometric method," Analytical Methods, vol. 4, no. 8, pp. 2283-2287, 2012.

[19] J. Yu, L. H. Zuo, H. J. Liu, L. J. Zhang, and X. J. Guo, "Synthesis and application of a chiral ionic liquid functionalized b-cyclodextrin as a chiral selector in capillary electrophoresis," Biomedical Chromatography, vol. 27, no. 8, pp. 1027-1033, 2013.

[20] Y. M. Lee, S. Y. Nam, and D. J. Woo, "Pervaporation of ionically surface crosslinked chitosan composite membranes for wateralcohol mixtures," Journal of Membrane Science, vol. 133, no. 1, pp. 103-110, 1997.

[21] Y. Matsuoka, N. Kanda, Y. M. Lee, and A. Higuchi, "Chiral separation of phenylalanine in ultrafiltration through DNAimmobilized chitosan membranes," Journal of Membrane Science, vol. 280, no. 1-2, pp. 116-123, 2006.

[22] J. H. Kim, J. H. Kim, J. Jegal, and K. H. Lee, "Optical resolution of $\alpha$-amino acids through enantioselective polymeric membranes based on polysaccharides," Journal of Membrane Science, vol. 213, no. 1-2, pp. 273-283, 2003.

[23] H. D. Wang, L. Y. Chu, H. Song, J. P. Yang, R. Xie, and M. Yang, "Preparation and enantiomer separation characteristics 
of chitosan/ $\beta$-cyclodextrin composite membranes," Journal of Membrane Science, vol. 297, no. 1-2, pp. 262-270, 2007.

[24] T. Pralhad and K. Rajendrakumar, "Study of freeze-dried quercetin-cyclodextrin binary systems by DSC, FT-IR, X-ray diffraction and SEM analysis," Journal of Pharmaceutical and Biomedical Analysis, vol. 34, no. 2, pp. 333-339, 2004.

[25] J. Wang, Y. P. Cao, B. G. Sun, and C. T. Wang, "Characterisation of inclusion complex of trans-ferulic acid and hydroxypropyl$\beta$-cyclodextrin," Food Chemistry, vol. 124, no. 3, pp. 1069-1075, 2011.

[26] L. X. Song, C. F. Teng, and Y. Yang, "Preparation and characterization of the solid inclusion compounds of $\alpha$-, $\beta$-cyclodextrin with phenylalanine (D-, L- and DL-Phe) and tryptophan (D-, Land DL-Trp)," Journal of Inclusion Phenomena, vol. 54, no. 3-4, pp. 221-232, 2006.

[27] W. H. Tang, I. W. Muderawan, T. T. Ong, and S. C. Ng, "Facile synthesis of positively charged monosubstituted $\alpha$ - and $\gamma$-cyclodextrins for chiral resolution of anionic racemates," Tetrahedron Asymmetry, vol. 18, no. 13, pp. 1548-1553, 2007.

[28] F. Veiga, J. J. C. Teixeira-Dias, F. Kedzierewicz, A. Sousa, and P. Maincent, "Inclusion complexation of tolbutamide with $\beta$ cyclodextrin and hydroxypropyl- $\beta$-cyclodextrin," International Journal of Pharmaceutics, vol. 129, no. 1-2, pp. 63-71, 1996. 

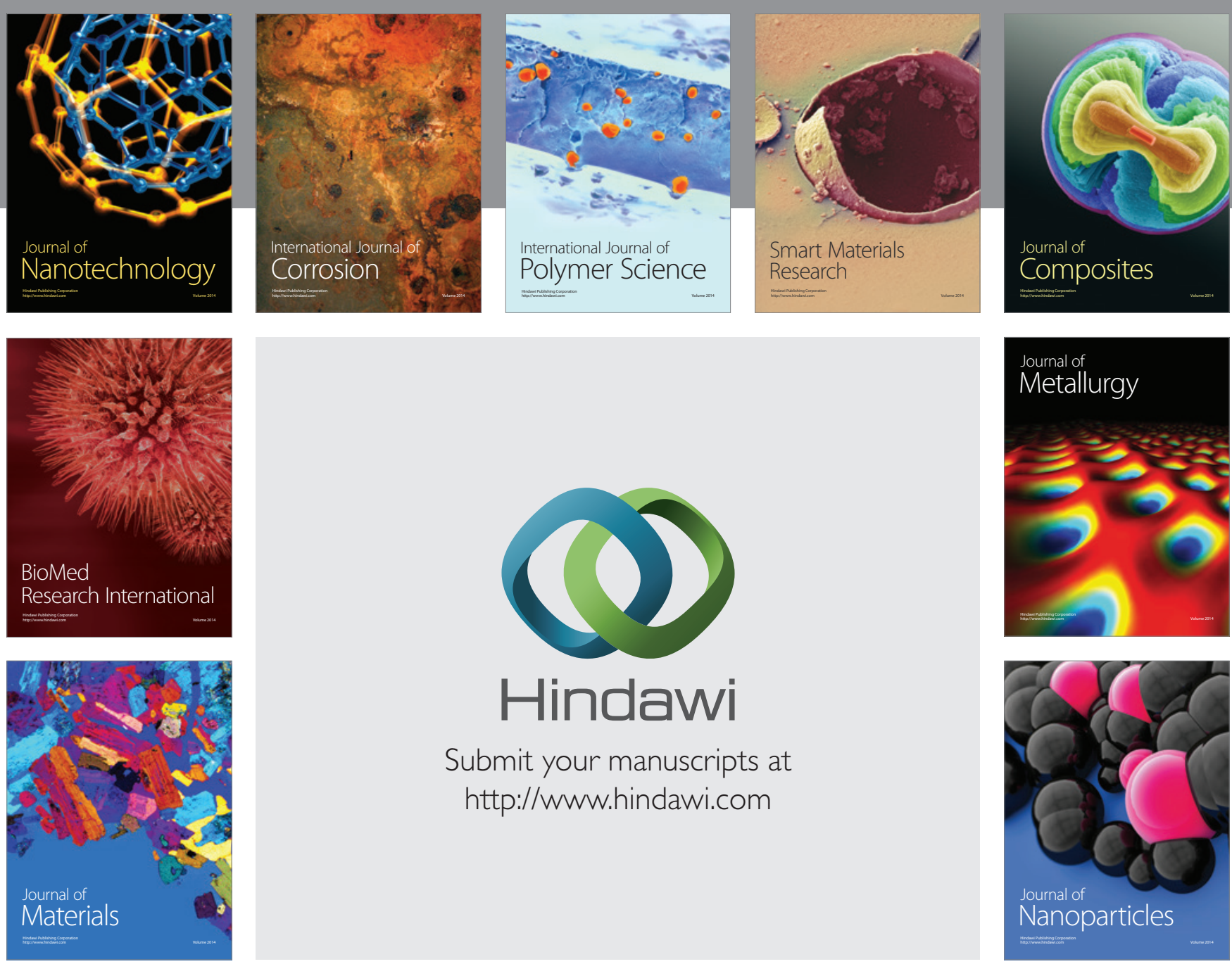

Submit your manuscripts at http://www.hindawi.com
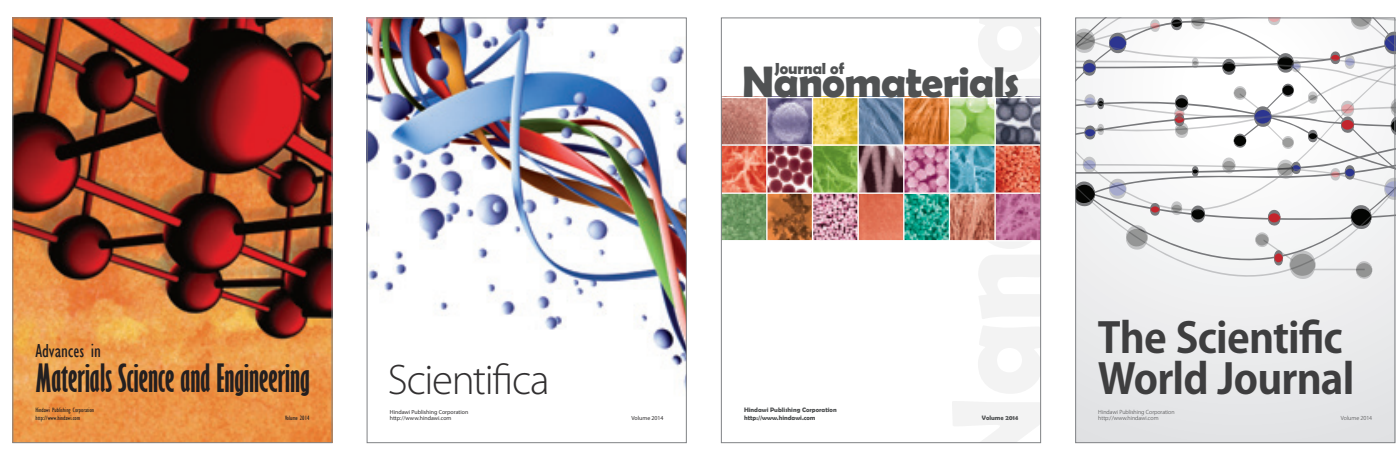

\section{The Scientific World Journal}
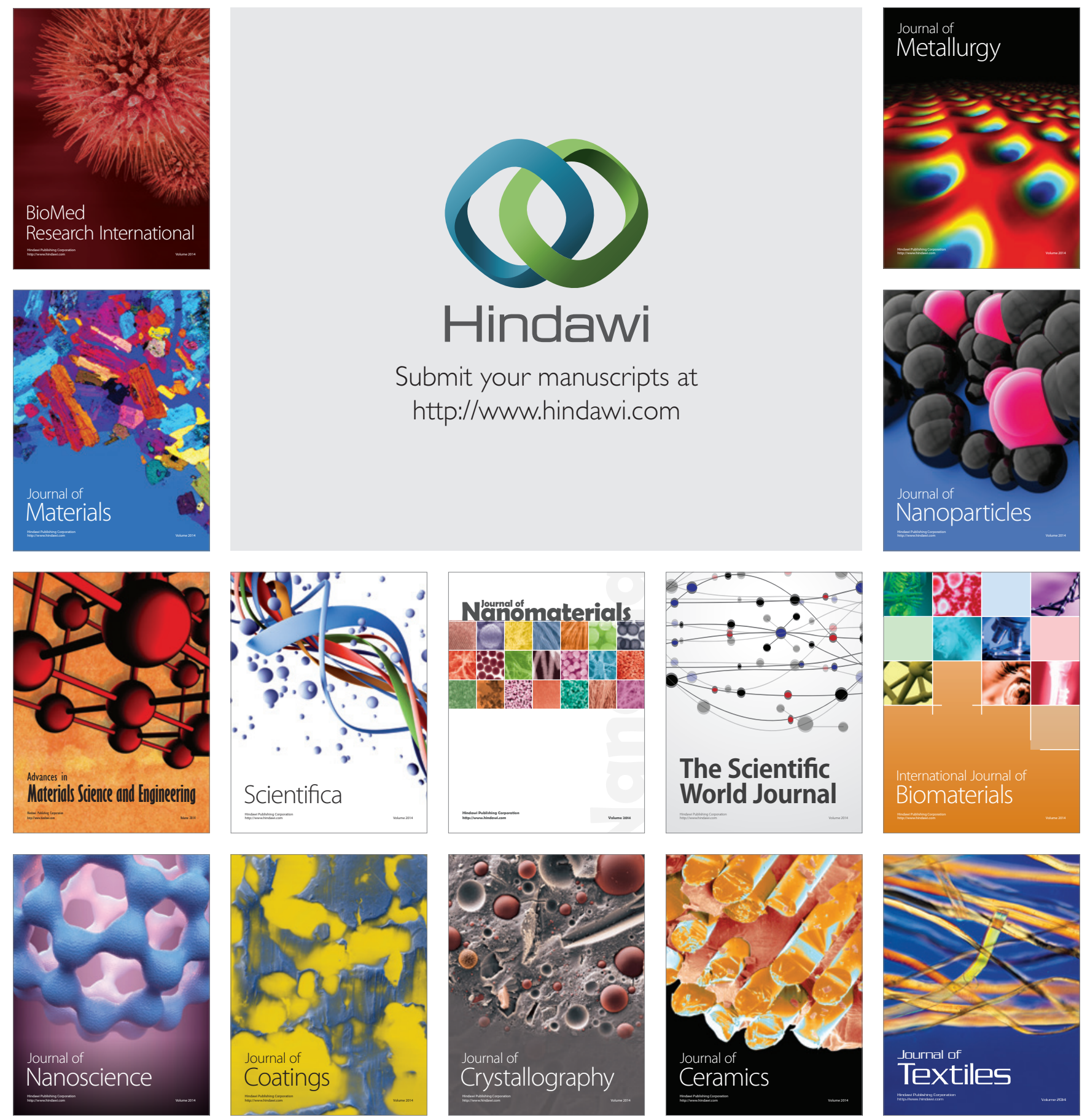